\title{
Desarrollo de tecnología para la fabricación DE MÁQUINAS CNC PARA CORTE DE TENDIDOS DE TELA PARA PEQUEÑOS TALLERES DE CONFECCIÓN
}

\author{
IVAN ARANGO ${ }^{1}$ \\ FABIO PINEDA ${ }^{2}$
}

\section{Resumen:}

Este artículo presenta el resultado de varias investigaciones relacionadas con el desarrollo de tecnología aplicada al diseño y la fabricación de máquinas $\mathrm{CNC}$ (control numérico por computador) para corte de tendidos de tela, destinadas a pequeños talleres de confección. El desarrollo responde a la necesidad de la industria de acceder a máquinas de producción fraccionada de bajos costos de operación. Desde diversos puntos de vista la solución encontrada presenta factores novedosos. El primero de ellos, la inversión de la dinámica de los tendidos de tela. Otro involucra el actuador de corte, el cual no se construyó a la medida de la máquina, sino que fue el resultado de un diseño para habilitar actuadores comerciales utilizados en el proceso manual. El equipo recibió el software CAD/CAM de otras investigaciones del mismo grupo. La máquina, por sus innovaciones, recibió derechos de la oficina de patentes de Colombia.

\section{Palabras clave:}

Diseño mecatrónico, confección, corte de telas, CNC, CAD-CAM.

1 Ingeniero Mecánico, M. Sc, Docente Universidad Eafit, iarango@eafit.edu.co

2 Ingeniero Mecánico, M. Sc, Docente Universidad Eafit, fpineda@eafit.edu.co 


\section{Abstract:}

This paper presents the results of several investigations related with the development of technology to design and manufacture of CNC machines, to cut fabric layers for small workshops. The development responds to the need for industry to access to fractional production machines with low operating costs. From various points of view, the solution has introduced new design factors. The first, the reversal of the dynamics of fabric layers. Another involves the cutting actuator, which was not built to suit the machine, but was the result of a design to enable the integration of commercial actuators used in manual processes. The equipment received the CAD/CAM software from other investigations in the same group. The machine, for its innovations, received rights from the patent office of Colombia.

\section{Key words:}

Mechatronic design, apparel, cut of fabrics, CNC, CAD-CAM. 


\section{INTRODUCCIÓN}

En la industria de la confección de prendas de vestir se han realizado grandes avances en todos los procesos que involucra. Algunos de los más significativos en lo que tiene que ver con la productividad y capacidad de innovación de esta industria son los siguientes: El diseño (patronado, escalado, trazado) pasó de realizarse mediante patrones manuales a la utilización de software CAD especializado (Arango, 1997). El corte está pasando de manual a computarizado. En el proceso manual se utilizan hojas impresas con las formas de las secciones a cortar, las cuales al ponerse sobre los tendidos de tela actúan como guías para los operarios. En el proceso computarizado se utilizan máquinas que tienen una mesa donde los tendidos se sujetan mediante vacío y el corte se efectúa con actuadores que van montados en mesas cartesianas con sistemas de movimiento XYC comandados desde un computador (Betancourt, 2004). Y en la unión (costura) de los componentes, además de la clásica máquina de coser se utilizan máquinas especializadas en funciones tales como unión de bolsillos, cuellos, bordados, etc.

Todos estos avances han permitido reducir los costos de producción de las prendas, especialmente cuando los volúmenes de fabricación son grandes. Esto ha propiciado la fundación de grandes centros de confección especializados en la producción de prendas clásicas o de uso casual, además de otras prendas que no son de vestir pero que se fabrican con tela como son las fundas de almohada, cubiertas de muebles, cortinas, etc.

Se esperaría que por el alto grado de especialización alcanzado, las fábricas de confección fueran características de países desarrollados. Sin embargo, hay razones para que esto no sea así. La confección de prendas aún tiene mucho de manual en el ensamble, transporte, revisión, estampado, planchado y empaque. Como consecuencia, la confección continúa siendo una actividad propia de países con mano de obra calificada y barata. A manera de ejemplo, en Colombia hay cerca de 450 fabricantes de textiles y 10000 confeccionistas, la mayoría de los cuales son considerados 
pequeños fabricantes. El 50\% tienen entre 20 y 60 máquinas y participan del 27\% en el PIB industrial del país. De estas, el $20 \%$ de las empresas confeccionistas está en Medellín (Cámara de Comercio de Medellín et al., 2006).

Existen otros factores que han impedido que la confección de prendas de vestir se convierta en un proceso completamente automatizado y con grandes volúmenes de producción. Los principales son la moda, la calidad, la marca (logo), el gusto de las personas por la variedad. Estos factores permiten que países ricos se hayan hecho muy prolíficos en generar moda y que posean industria de confección, pero con talleres no muy grandes ubicados en países con mano de obra barata.

Los desarrollos industriales han tocado a todas las operaciones de confección tales como el bordado, que involucra máquinas con una o varias estaciones, o el patronado y escalado que utiliza software CAD-CAM (Villa \& Jaramillo, 2005). El corte también se ha beneficiado con la inclusión de máquinas CNC pero diseñadas para grandes volúmenes de producción. A manera de ejemplo se puede ver que máquinas pequeñas en esta operación como las Lectra Systems ${ }^{\circledR}$ referencia Vector2500 o Gerber ${ }^{\circledR}$ referencia GT3250, tienen velocidades de corte promedio de $10 \mathrm{~m} / \mathrm{min}$ (incluyendo las paradas para carga y otras). Ambas máquinas pueden cortar $25 \mathrm{~mm}$ de tendidos de tela simultáneamente (equivalentes a 50 tendidos de tela relativamente gruesa). Si se tratase de camisas, como el perfil de corte de estas no sobrepasa los $5 \mathrm{~m}$, se pueden cortar alrededor de 100 camisas por minuto, lo que es equivalente a la producción diaria de un taller pequeño. Esto sugiere que la operación de corte se puede convertir en una parte del proceso de confección, a la cual el pequeño taller puede acceder a través de un tercero que solo realiza esta función para muchos talleres. Sin embargo, problemas de transporte, disponibilidad y el que estas máquinas son costosas tanto en inversión como en operación, indican que es necesario diseñar equipos con un nivel de producción inferior, del orden de 1 a 5 prendas por minuto, con un costo de inversión y operación proporcional a la reducción en velocidad, sin 
eliminar características positivas como flexibilidad y calidad que son diferenciadoras para competir en los mercados internacionales, como lo indican las investigaciones (Bernal \& Ruiz, 1999).

En el proceso para diseñar prendas de vestir, se deben realizar varios pasos antes de producir una prenda (Ocampo, 2004). En forma resumida estos pasos son: Diseño, el cual incluye patronaje y escalado. Corte, que incluye el tendido de la tela, el trazado ordenado (sólo en el proceso manual) y el corte. Costura o unión de los diferentes componentes de la prenda. Y acabado, que incluye planchado, etiquetado, empaque (en algunos casos también el teñido y el estampado o bordado).

Se reconocen actualmente dos formas de realizar estos pasos: una tradicional en pequeños talleres sin tecnología moderna, que se puede denominar "producción manual", y otra en la que algunos de estos pasos se han sistematizado aumentando la flexibilidad y el tiempo de respuesta ante cambios en la moda, disminuyendo significativamente el tiempo de proceso y los respectivos costos asociados (Arango, 2004). Esta segunda forma se puede denominar "producción sistematizada".

La producción sistematizada utiliza 2 tecnologías complementarias que permiten realizar el diseño y el corte del producto. Estas son el Diseño Asistido por computador (CAD) y la Manufactura Asistida por Computador (CAM) (Villa \& Jaramillo, 2005).

En el proceso manual, se denominan patrones a las figuras hechas en cartón grueso que tienen la forma de una sección perteneciente a una prenda. Ejemplos de patrones son el espaldar, el cuello, los bolsillos o la manga de una camisa. Para una prenda en particular se tienen por lo tanto varios patrones. Estos se utilizan para trazar lo más eficientemente posible la tela antes de cortarla. Para cada una de las tallas de un tipo de prenda se debe disponer de un juego apropiado de patrones. El proceso de asignación de las diferentes tallas a partir de una medida de referencia se denomina escalado. Es obvio que el proceso de diseño y producción involucra un gran número de patrones, lo que implica un sistema de identificación, clasificación y almacenamiento adecuado para su 
manejo. Por último, el corte se realiza sobre una mesa en la cual se disponen de uno a cien tendidos de tela. Se utiliza una cortadora manual, que el operario dirige sobre las figuras que deben cortar.

En el proceso sistematizado, los pasos mencionados se realizan en un programa que incluye funciones que ayudan a realizar el trabajo mucho más eficazmente. En este caso, el diseño, organización y almacenamiento de los patrones es virtual. La maximización de las secciones a cortar (nestling) se realiza en un ambiente computacional. En el proceso de corte, el movimiento del actuador lo realiza un robot cartesiano comandado desde un computador.

\section{Métodos}

Para el desarrollo de los objetivos que conducen a resolver la necesidad planteada en esta investigación, se escogió como soporte el diseño metódico con sus extensiones para ingeniería mecánica (VDI2221, 1993) e ingeniería mecatrónica (VDI2206, 1993). Algunos de los pasos más significativos de esta metódica aplicados a este trabajo se listan a continuación.

\subsection{Definición de producto}

La cortadora de tela $\mathrm{CNC}$, es una máquina dedicada a cortar automáticamente varias capas de tela de diferentes formas, pesos, calidades y espesores. Es necesario tener precisión en el corte, repetitividad en el proceso, optimizar el recurso humano y minimizar costos, defectos y pérdidas de material. Es un equipo aplicable también a otro tipo de materiales como cuero y nuevos materiales compuestos (Gallo, 2005).

\subsection{Clarificación de los objetivos del diseño}

Para llevar a cabo la fabricación de la cortadora de tela automática, se requiere diseñar toda la estructura de soporte y los diferentes subsistemas de control de movimiento, sujeción y corte de los tendidos de tela. Para un diseño bajo requerimientos no estandarizados como es el caso, además de las demandas específicas, 
se debe cumplir con unos objetivos normales de diseño, que fueron definidos en las siguientes categorías: seguridad, confiabilidad de operación, facilidad de uso y mantenimiento. A cada una de estas categorías, se le definen los objetivos que se desean lograr. Estos se muestran en la Tabla 1.

Tabla 1. Objetivos de diseño

\begin{tabular}{llll}
\hline \multicolumn{1}{c}{ Seguridad } & $\begin{array}{c}\text { Confiabilidad en } \\
\text { operación }\end{array}$ & \multicolumn{1}{c}{$\begin{array}{c}\text { Facilidad } \\
\text { de uso }\end{array}$} & $\begin{array}{c}\text { Facilidad de } \\
\text { mantenimiento }\end{array}$ \\
\hline $\begin{array}{l}\text { Bajo riesgo de } \\
\text { desprendimiento } \\
\text { de partes }\end{array}$ & $\begin{array}{l}\text { Bajo desgaste de } \\
\text { partes móviles }\end{array}$ & $\begin{array}{l}\text { Facilidad para } \\
\text { poner en marcha }\end{array}$ & Fácil lubricación \\
$\begin{array}{l}\text { Bajo riesgo de } \\
\text { cortes y lesiones }\end{array}$ & $\begin{array}{l}\text { Buena resolución } \\
\text { en sistemas de } \\
\text { posicionamiento }\end{array}$ & $\begin{array}{l}\text { Software intuitivo, } \\
\text { fácil de utilizar }\end{array}$ & $\begin{array}{l}\text { Mínimo número de } \\
\text { componentes }\end{array}$ \\
$\begin{array}{l}\text { Bajo riesgo } \\
\text { de accidentes } \\
\text { eléctricos }\end{array}$ & $\begin{array}{l}\text { Poca frecuencia } \\
\text { de calibración de } \\
\text { partes }\end{array}$ & $\begin{array}{l}\text { Ergonomía } \\
\text { adaptada al } \\
\text { operario local }\end{array}$ & $\begin{array}{l}\text { Facilidad de ensam- } \\
\text { ble o desensamble }\end{array}$ \\
$\begin{array}{l}\text { Facilidad de } \\
\text { paradas de } \\
\text { emergencia }\end{array}$ & $\begin{array}{l}\text { Mínimas } \\
\text { vibraciones }\end{array}$ & $\begin{array}{l}\text { Accesibilidad para } \\
\text { carga y descarga }\end{array}$ & $\begin{array}{l}\text { Diseño por módulos } \\
\text { recambiables }\end{array}$ \\
\hline
\end{tabular}

\subsection{Establecimiento de funciones}

La formulación de la función total de un sistema técnico consiste principalmente en llevar el objetivo del sistema por desarrollar, descrito verbalmente en una formulación de tareas, a una relación entre magnitudes de entrada y salida. Aquí es importante diferenciar entre flujos de energía, masa e información. En este caso, la función principal es cortar tendidos de tela (flujo de material) para formar secciones cortadas que forman parte de una prenda (Fig. 1).

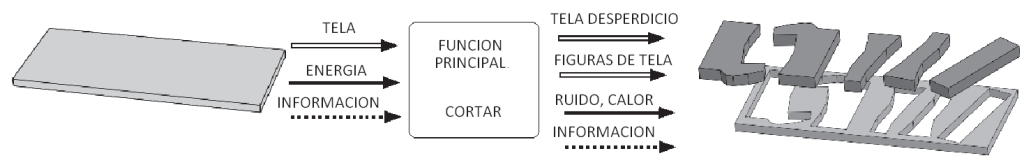

FIG. 1. DIAGRAMA DE CAJA NEGRA 
La materia ingresa en la máquina y sale dividida en secciones aprovechables, además de desperdicio. La información de entrada corresponde principalmente al dibujo de los perfiles de las figuras. La energía, eléctrica en este caso, se convierte en mecánica mediante motores y se utiliza en mover relativamente el actuador de corte con respecto a la tela. También se utiliza para mover alternativamente y a alta velocidad la cuchilla del actuador de corte. En la conversión de energía parte de ésta se convierte en calor. El movimiento y contacto de los diferentes elementos mecánicos genera ruido.

\subsection{División en sub-funciones}

El siguiente paso consiste en dividir la función general en sub-funciones o subsistemas. Algunas de las más importantes son las siguientes: Acoplar y sincronizar los sistemas eléctricos y mecánicos con el software; poner los tendidos de tela en la mesa de la máquina; sujetar los tendidos de tela para que no se muevan por acción de la fuerza de corte entre la cuchilla y la tela; cortar la tela de acuerdo con la forma de las secciones de las prendas que se van a confeccionar, este movimiento se realiza en un plano, por lo que se necesita descomponer el recorrido en dos direcciones, y por medio de interpolación obtener un movimiento controlado en cualquier dirección (Mejía, 2001); controlar el giro del actuador respecto a la tela debido a que las cuchillas de corte tienen un ancho y sólo cortan en una dirección, el actuador de corte es un subsistema completo que en los requerimientos se indica que debe ser un cortador manual; y desplazar horizontalmente los tendidos cortados, para permitir el acceso de tendidos frescos.

$\mathrm{Al}$ dividir la función de la máquina en sub-funciones, se puede visualizar de una manera más clara que es lo que sucede dentro de la caja negra. Esto quiere decir que desde un punto de vista funcional, se pueden visualizar los flujos en forma de una estructura funcional también denominada caja transparente, en la cual se describe lo mejor posible lo que sucede con cada uno de los flujos para alcanzar los objetivos planteados en la función principal (Fig. 2). 


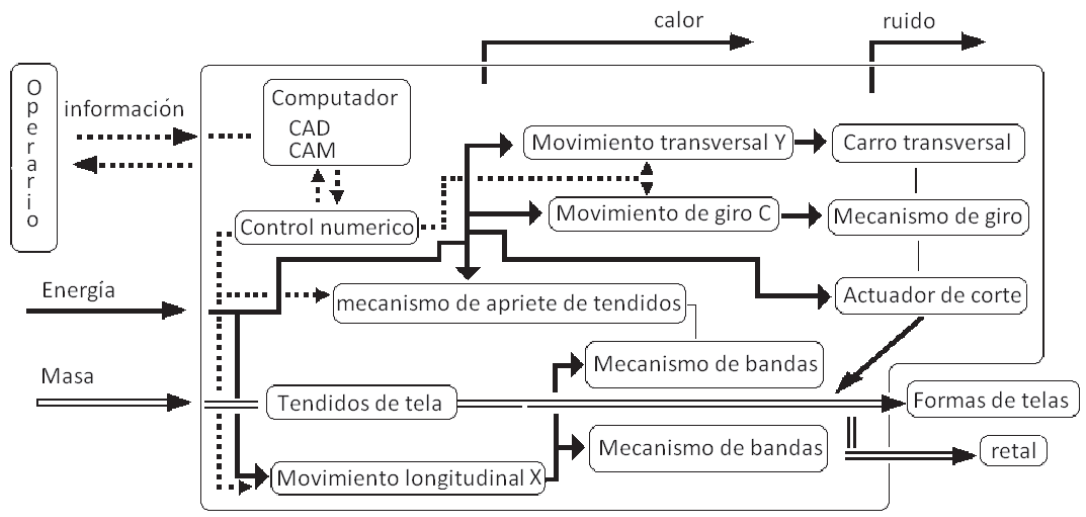

FIG. 2. DIVISIÓN DE LA MÁQUINA EN SUBSISTEMAS

En la metodología del diseño metódico se sugiere que el sistema se debe dividir en subsistemas ( $\left.\mathbf{S}^{1} \_\mathbf{S}\right)$, luego en sub-sub-sistemas ( $\left.{ }^{2} \_S\right)$ y así sucesivamente hasta llegar a la configuración más básica. Con el resultado se buscan varias opciones de solución para cada sub-,...,-sub-sistema ( $\mathrm{S}_{-}^{\mathrm{i}} \mathrm{S}$ ), de tal forma que al combinar las posibilidades se presenten muchas soluciones. Aquí sólo se llevará hasta la segunda escala, es decir, la de los subsistemas $\mathrm{S}^{2} \_\mathrm{S}$.

\subsection{Lista de requerimientos}

La lista de requerimientos pretende establecer límites precisos al diseño. Las especificaciones del producto también se conocen con el nombre de PDS (Product Design Specifications). En este caso los requerimientos más relevantes se presentan en la Tabla 2 . 
TABLA 2. LISTA DE REQUERIMIENTOS

\begin{tabular}{lcc}
\hline Requerimiento & Demandado & Deseado \\
\hline $\begin{array}{l}\text { Dimensiones. 3mx2,5mx2m máximo } \\
\text { Capacidad. Hasta 5 prendas por minuto }\end{array}$ & $\mathrm{X}$ \\
$\begin{array}{l}\text { Costo. Inferior a US } \$ 50.000 \text {. Este dato se obtuvo a } \\
\text { partir de una encuesta realizada a medianos y pequeños } \\
\text { confeccionistas de Medellín e Itagüí (Bernal, 1999). }\end{array}$ & $\mathrm{X}$ & \\
$\begin{array}{l}\text { Velocidad. Velocidad promedio no superior a un metro } \\
\text { por minuto (1 m/min). Este dato se toma del valor central } \\
\text { de la producción tabulada en una distribución estándar. }\end{array}$ & \\
$\begin{array}{l}\text { Costo de operación. El consumo de energía no debe } \\
\text { superar 40kW turno/hora. Sin consumo de aire. }\end{array}$ & $\mathrm{X}$ & $\mathrm{X}$ \\
$\begin{array}{l}\text { Corte. Debe adaptarse a partir de un actuador de corte } \\
\text { manual (comercial). }\end{array}$ & $\mathrm{X}$ & \\
$\begin{array}{l}\text { Infraestructura eléctrica. Máximo 5kW (bifásica, a 220V) } \\
\text { Software. Desarrollo propio. }\end{array}$ & $\mathrm{X}$ & $\mathrm{X}$ \\
\hline
\end{tabular}

\subsection{Alostracción para identificar los problemas esenciales}

En un nuevo diseño se comparan las especificaciones con respecto a las de equipos conocidos. Las diferencias en características (deltas) determinan la dificultad del diseño. Cuando todos los requerimientos tienen deltas negativos, sólo es posible obtener un diseño con tecnologías costosas. En este caso, la velocidad presenta un delta positivo y por ello se analizan los efectos de su variación en la variación de los requerimientos que presentan deltas negativos como son el costo y el consumo de energía.

A continuación se analizan los sub-sistemas relacionados con los requerimientos. Todos los subsistemas sufren modificaciones con el cambio de los demás. Solo se describen los tres subsistemas asociados con los requerimientos obligatorios.

\subsubsection{Sub-sistema de sujeción de tendidos de tela}

En el análisis de costos de operación se encontró que en los modelos comerciales el mayor costo se debe al consumo de energía 
del sistema de sujeción. Este está constituido por una turbina que genera vacío sobre la mesa donde se extienden los tendidos (Fig. 3). La mesa dispone de pequeños agujeros por donde ingresa el aire. Para mejorar la sujeción sobre los tendidos se extiende una hoja de papel con agujeros. Algunas máquinas, para disminuir el consumo de energía han incorporado vacío por sectores. Aun así, el consumo en máquinas pequeñas es superior a $15 \mathrm{~kW} / \mathrm{hr}$.

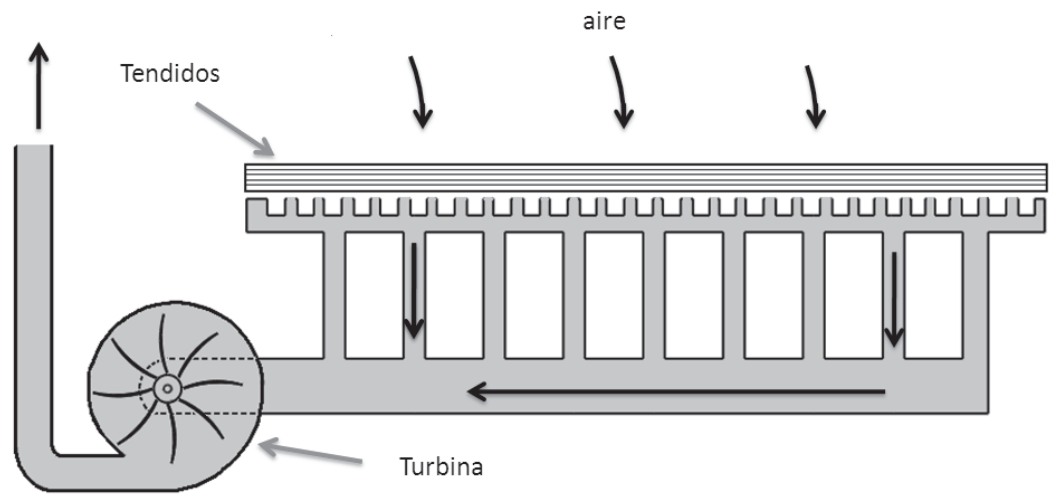

FIG. 3. SUJECIÓN ESTÁNDAR DE TENDIDOS DE TELA

Para reemplazar este subsistema se analizan varias opciones de sujeción. Como variables relacionadas con la sujeción de los tendidos se debe tener en cuenta que: El espesor y el número de tendidos de tela varía; no se puede interponer ningún elemento en el recorrido de la cuchilla de corte, y la sujeción y liberación deben ser rápidas y sin deteriorar la tela.

El método exige que para cada sub-sistema se presenten varias propuestas de forma que se puedan combinar o permutar para generar varias alternativas que luego se califican con factores relacionados con los requerimientos. Para el caso se analizaron diferentes mecanismos de sujeción (Rodríguez \& Pineda, 2009). Algunos de estos son: prensas mecánicas o magnéticas, pinzas, alicates, cuerdas, alambres, tornillos, clavos y vacío como ya se explicó (Fig. 4). 

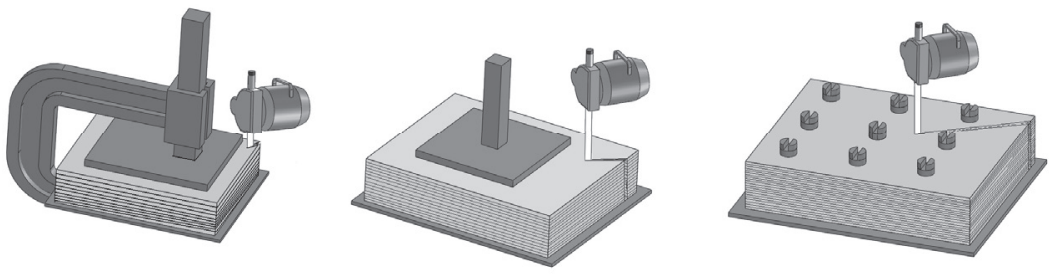

Fig. 4. Soluciones a LA FUnCIÓn DE SUJETAR LOS TENDIDOS DE TELA

El siguiente análisis consiste en calificar las alternativas presentadas para resolver la función de sujetar. En este caso se consideran como factores importantes el costo y el consumo de energía (Tabla 3).

TABLA 3. CalificACIÓN DE OPCIONES

\begin{tabular}{lllc}
\hline \multicolumn{1}{c}{ Tipo } & \multicolumn{1}{c}{ Ventajas } & Desventajas & C. energía \\
\hline Prensa magnética & $\begin{array}{l}\text { La presión es } \\
\text { uniforme }\end{array}$ & $\begin{array}{l}\text { Interfiere con el } \\
\text { proceso de corte }\end{array}$ & Alto \\
$\begin{array}{l}\text { Prensa mecánica, } \\
\text { pinzas, alicates }\end{array}$ & Simple, conocido & $\begin{array}{l}\text { Interfiere con el } \\
\text { proceso de corte }\end{array}$ & Bajo \\
Clavos, tornillos & Fácil manipulación & Deteriora la tela & Bajo \\
\hline
\end{tabular}

El resultado apunta a utilizar la prensa mecánica resolviendo la desventaja planteada. La prensa debe tener una zona pequeña en la que pueda trabajar la cuchilla. Esta solución obliga a que sean los tendidos de tela los que se muevan. Para lograrlo, la tela se sujeta mediante grupos de bandas superiores e inferiores. Estas bandas son movidas por un servomotor, dando como resultado que el tendido se desplace en la dirección X con alta precisión utilizando potencias inferiores a $1 \mathrm{~kW}$ (Fig. 5). 


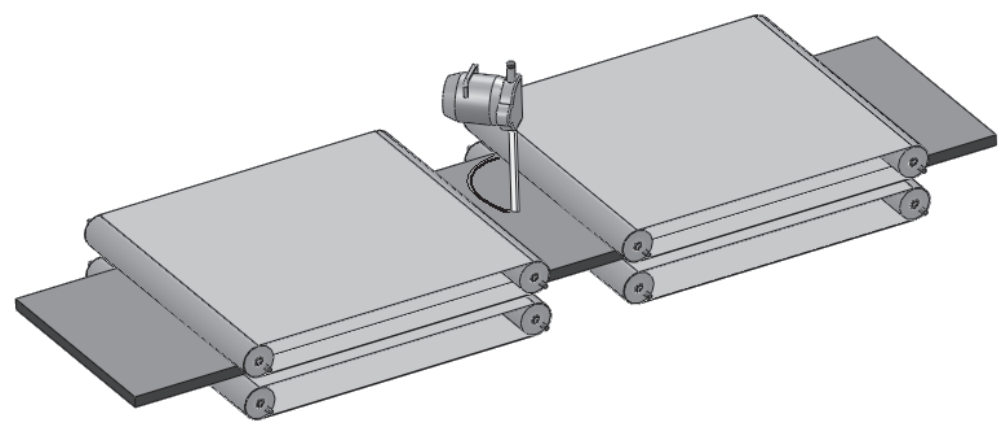

Fig. 5. SOLUCIÓN PARA SUJECIÓN DE TENDIDOS DE TELA

Las bandas superiores tienen un mecanismo que les permite el desplazamiento en la dirección vertical, es decir, pueden disminuir su distancia con el fin de sujetar el tendido de tela. El mecanismo consta de unos tornillos que son girados por engranajes que reciben movimiento por cadena de un motor común. Las tuercas están fijas a la estructura que soporta las bandas superiores y al girar los tornillos se desplazan moviendo las bandas.

\subsubsection{Sulb-sistema de corte}

Por exigencia de diseño (requerimientos), se utilizó un actuador de corte comercial. Por lo tanto, el diseño se enfocó en soportarlo, hacerlo girar y moverlo a lo largo del eje Y. En la dirección X, el actuador se ubica entre las bandas anteriores y posteriores.

Se presentan dos problemas en la zona de corte. El primero, durante el movimiento de avance y retroceso de la tela y a medida que se corta, las bandas dejan caer los extremos de los tendidos inferiores entre las bandas (Fig. 6). El segundo, la punta de la cuchilla del actuador de corte debe atravesar los tendidos para que el corte quede limpio y los tendidos del fondo queden cortados. Para que los extremos de los tendidos no caigan en la zona de corte se diseñó una banda que también tiene la función de proteger el extremo de la cuchilla del actuador de corte y garantizar que la tela no se enrede. 


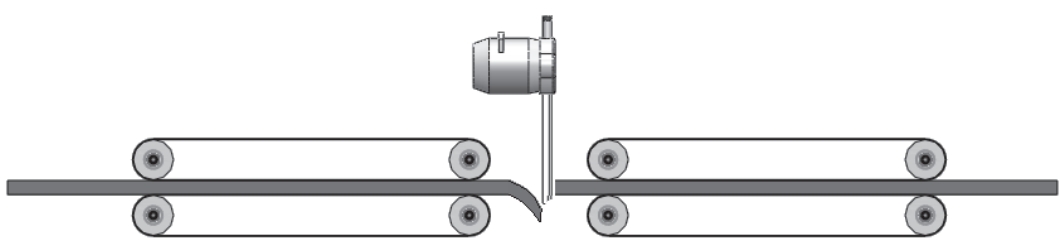

Fig. 6. Problemas En LA zona de CORTE

El desplazamiento de esta banda es en sentido transversal y obtiene el movimiento mediante una transmisión que viene desde el servomotor Y (Fig. 7). En la banda se encuentra un mecanismo llamado recibidor que está constituido por un disco excéntrico que se atornilla a la tuerca que hace parte de todos los cortadores comerciales y que va soldada al protector de la cuchilla. Cuando el actuador de corte gira también gira la cuchilla, el protector de cuchilla, la tuerca y la excéntrica. El resultado es que el filo de la cuchilla se convierte en el eje de giro de todo el sistema garantizando un corte bien orientado (Fig. 7, lado derecho).
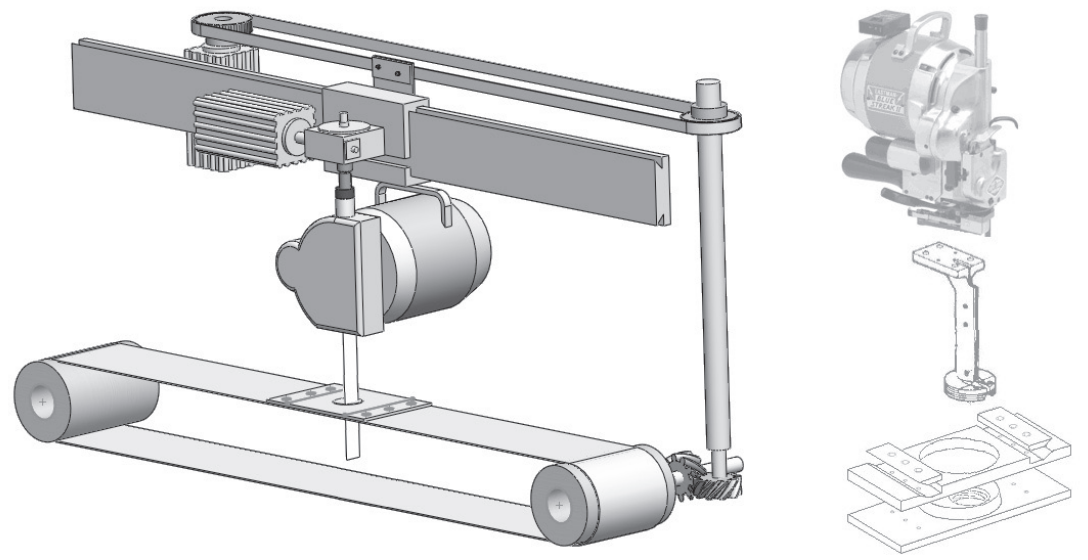

FIG. 7. SUB-SISTEMA DE CORTE

\subsubsection{Sub-sistema de movimiento}

Todos los movimientos son de precisión, con retroalimentación. A este sub-sistema ingresa energía eléctrica e información de la 
posición deseada como se muestra en el diagrama de flujo de la Fig. 8. El control evalúa la diferencia entre la posición actual y la deseada, e introduce el error en un algoritmo PID. El resultado es una orden para un administrador de energía que de acuerdo al valor, permite el paso de mayor o menor cantidad de energía hacia el convertidor (motor) y de ahí la energía mecánica va hacia el mecanismo, que en este caso puede ser el eje X o Y, o el eje de giro del actuador.

\subsubsection{Sub-sistema de información}

El software CAM seleccionado para adaptarlo a la máquina provino de una investigación precedente en la que se desarrolló una aplicación genérica de forma que se pudiese adaptar a cualquier máquina (Arango, 1998). El software cursor CAM está desarrollado con base en ventanas.

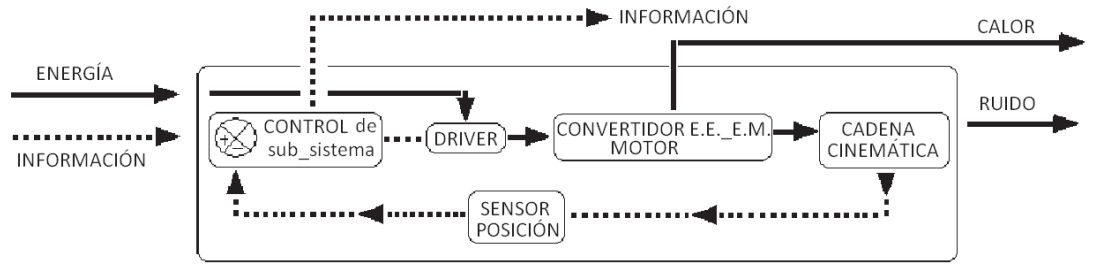

Fig. 8. Diagrama de los Subsistemas de movimiento

En estas se pueden realizar actividades relacionadas con la configuración, el ajuste, el trabajo de corte, el diagnóstico de todos los subsistemas y el archivo de trabajos realizados. Igualmente, se utilizó un software $\mathrm{CAD}$ desarrollado por estudiantes de ingeniería mecánica en su proyecto de grado (Villa \& Jaramillo, 2005). El equipo también admite diseños desarrollados en otras aplicaciones $\mathrm{CAD}$ mediante código $\mathrm{G}$ normal o a través de post-procesadores.

\section{Resultados}

con base en la información recopilada en la lista de requerimientos y considerando las opciones que resultan de analizar los 
diferentes subsistemas, se propone el diseño final de la cortadora mostrado en la Fig. 9 (Eafit, 2004). Los dos requerimientos principales solicitados se cumplieron: el valor del equipo y el consumo de energía. Por motivos de espacio, no se presenta en este documento el detalle de las características de todos los componentes de la máquina (rodamientos, engranes, actuadores, motores, anclajes, soldaduras, etc.). Pero, para efectos de comparación, en la Tabla 4 se presentan las características de la máquina con respecto a dos equipos comerciales comúnmente encontrados en la industria. El sistema de sujeción se probó con varios tipos de tendidos observando su buen funcionamiento con diferentes espesores y texturas, conservando su acabado sin inducir arrugas en las telas.

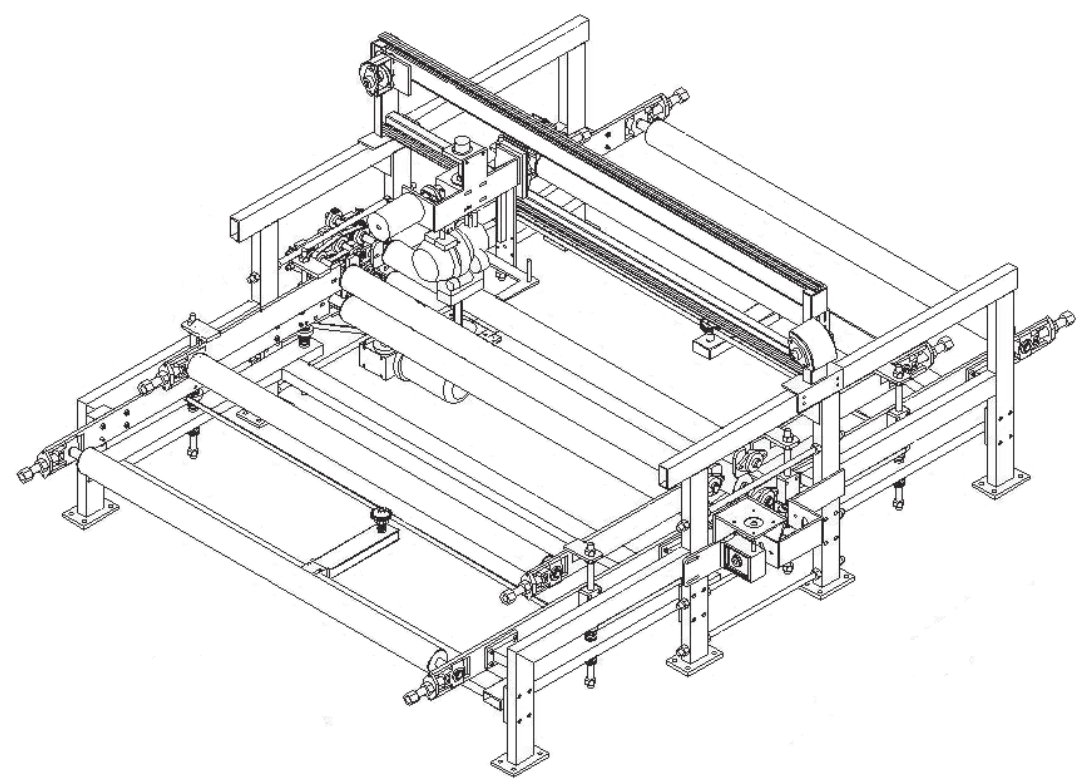

Fig. 9. Esquema general de Cortadora CNC de tendidos de tela 
TABLA 4. Comparación de LA MÁQUINA CON EQUIPOS COMERCIALES

\begin{tabular}{lccc}
\hline \multicolumn{1}{c}{ Característica } & GT®3250 & Vector® 2500 & Diseño \\
\hline Altura material comprimido & $3,2 \mathrm{~cm}$ & $2,5 \mathrm{~cm}$ & $5,0 \mathrm{~cm}$ \\
Ancho útil de corte & $170 / 200 / 240 \mathrm{~cm}$ & $175 \mathrm{~cm}$ & 150 \\
Largo útil de corte & $170 / 254 \mathrm{~cm}$ & $180 \mathrm{~cm}$ & 180 \\
Velocidad máxima de corte & $46 \mathrm{~m} / \mathrm{min}$ & $100 \mathrm{~m} / \mathrm{min}$ & $2 \mathrm{~m} / \mathrm{min}$ \\
Rendimiento & $9,5 \mathrm{~m} / \mathrm{min}$ & $\mathrm{NA}$ & $1,0 \mathrm{~m} / \mathrm{min}^{\circledR}$ \\
Aceleración cabeza corte & $3,7 \mathrm{~m} / \mathrm{sec}^{2}$ & $1,0 \mathrm{~m} / \mathrm{sec}^{2}$ & $0,3 \mathrm{~m} / \mathrm{sec}^{2}$ \\
Dimensiones de máquina & $234 \times 437 \times 91$ & $280 \times 380 \times 108$ & $300 \times 220 \times 140$ \\
Peso de máquina & $2275 \mathrm{Kg}$. & $2500 \mathrm{~kg}$ & $1450 \mathrm{~kg}$ \\
Potencia de la turbina & $30 \mathrm{~kW}$ & $15 \mathrm{~kW}$ & $1 \mathrm{~kW}(\mathrm{sujeción})$ \\
Potencia unidad de control & $12 \mathrm{~kW}$ & $6,5 \mathrm{~kW}$ & $3 \mathrm{~kW}$ \\
Potencia consumo promedio & $18 \mathrm{~kW}$ & $\mathrm{NA}$ & $3,2 \mathrm{~kW}$ \\
Consumo de aire & $11,3 \mathrm{lpm}(6,8 \mathrm{bar})$ & $50 \mathrm{lpm}(6 \mathrm{bar})$ & 0 \\
Nivel de ruido & $75 \mathrm{~dB}$ & $75 \mathrm{~dB}$ & $72 \mathrm{~dB}$ \\
\hline
\end{tabular}

El desempeño mecánico y electrónico de la cortadora de tela CNC (Fig. 10) es satisfactorio y acorde con lo esperado según los requerimientos del diseño. El actuador de corte rotativo trabaja bien con telas normales, sin embargo es necesario realizar pruebas adicionales de corte para determinar el comportamiento con materiales menos utilizados. El calentamiento de la cuchilla durante las pruebas de corte no excede los parámetros de trabajo estipulados por el fabricante. Si no se ajustan con la presión adecuada el tendido tiende a arrugarse ya que se generan movimientos relativos entre capas de tela (Bonil, 2005). 


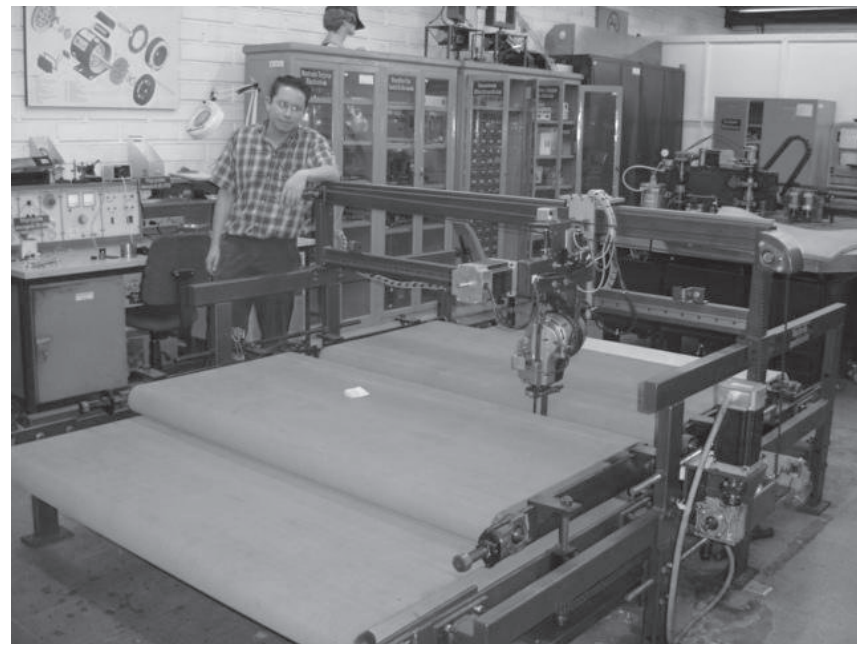

FIG. 10. MÁQUINA CORTADORA EN PROCESO DE PRUEBAS

\section{Discusión}

El proceso de diseño de maquinas fraccionarias para sectores productivos pequeños con relación a los mercados globales tiene un gran potencial y grandes retos que vencer. Es rentable siempre y cuando adicional a lo fraccional se anexe un diferenciador en la calidad, innovación en el producto o en lo económico.

En el equipo diseñado se logró resolver un problema de consumo de energía al mismo tiempo que se redujo el precio general del equipo. También se resolvió una barrera tecnológica al utilizar actuadores comerciales de corte en vez de diseñar y construir una parte del equipo muy dispendiosa tecnológicamente. Por sus innovaciones en la solución de estos problemas, la máquina recibió derechos de la oficina de patentes de Colombia (SIC, 2004).

\section{Conclusiones}

Las modificaciones iníciales al software CAM para la operación de la cortadora de tela CNC son exitosas. Los diferentes módulos 
que posee el software son adaptables a cada función del equipo gracias a que sus características son similares a los sistemas de arquitectura abierta. El espesor del arreglo de material a trabajar no es un factor importante en el desempeño del equipo. Es necesario continuar con las pruebas de corte en ambiente de producción, ya que es necesario conocer el comportamiento de los diferentes componentes del equipo electrónico y mecánico en condiciones de trabajo como las encontradas en la industria de la confección colombiana.

Las geometrías de patrones complejos, en las cuales se pueden encontrar ángulos agudos, exigen modificaciones en el proceso $\mathrm{CAD}$, en la fase de diseño para evitar que el actuador rotativo deforme los tendidos al tratar de seguir esta geometría.

\section{Referencias}

Arango, I., (1997); From the drawing to the actual piece, inside CAD/CAM programs, example of the textile industry. [Del dibujo a la pieza real, adentro de los programas CAD/CAM, ejemplo de la industria textil]. En memorias del XIII international congress on CAD/CAM, robotics and factories of the future, Pereira, Colombia.

Arango, I., (1998); Desarrollo de un software CAD/CAM plataformas para trabajar máquinas CNC manufactureras, Informe de investigación, Laboratorio Mecatrónica, Universidad Eafit, Medellín, Colombia.

Arango, I., (2004); Construcción y puesta a punto de una cortadora de tendidos de tela CNC, Informe de investigación, Laboratorio Mecatrónica, Universidad Eafit, Medellín, Colombia.

Bernal, E., Ruiz, J., (1999); Informe de visitas a industrias de la confección. Manuscrito no publicado, Universidad Eafit, Medellín, Colombia.

Betancur, S.C., (2004); Construcción, ensamble y puesta a punto de un actuador de corte para una máquina cortadora de tela, Proyecto de grado ingeniería mecánica, Laboratorio Mecatrónica, Universidad Eafit, Medellín, Colombia.

Bonil, L., (2005); Desarrollo de algoritmos para cortadoras de tela CNC, Especialización en diseño mecánico, Laboratorio Mecatrónica, Universidad Eafit, Medellín, Colombia. 
Cámara de Comercio de Medellín, Alcaldía de Medellín, Agencia de Cooperación internacional de Medellín, (2006); Manual para invertir en el sector textil-confección. Medellín, Colombia.

Eafit, (2004); Manual Cursor CAM 2, Manuscrito no publicado, Laboratorio de Mecatrónica, Universidad Eafit, Medellín, Colombia.

Gallo, A., (2005); Actuador CNC de corte de tipo cuchilla para cartón y cuero, Proyecto de grado ingeniería mecánica, Laboratorio Mecatrónica, Universidad Eafit, Medellín, Colombia.

Mejía, J.M. (2001); Desarrollo de interpoladores cartesianos para máquinas CNC, Proyecto de grado ingeniería mecánica, Laboratorio Mecatrónica, Universidad Eafit, Medellín, Colombia.

Ocampo, E., (2004); Diseño de una cortadora de tela automatizada, Proyecto de grado, Especialización en diseño mecánico, Laboratorio de Mecatrónica, Universidad Eafit, Medellín, Colombia.

Pineda, L., Jara, M., (2010); Prospectiva y Vigilancia Tecnológica en la Cadena Fibra-Textil-Confecciones, Bogotá, Colombia: Editorial Universidad del Rosario, Facultad de Administración.

Rodríguez, A., Pineda, F., (2009); Mecanismos en aparatos, máquinas e instrumentos. Medellín, Colombia: Editorial Eafit.

SIC, (2004); Superintendencia de Industria y Comercio, Cortadora automática de tendidos de telas. Solicitudes de patentes de modelo de utilidad, Gaceta No. 557, 523, Oficio 5374 de 02/06/2004.

VDI2206, (1993); Entwicklungsmethodik für mechatronische Systeme. [ Metodología de desarrollo para el diseño de sistemas mecatrónicos], Berlín, Alemania: Beuth Verlag.

VDI2221, (1993); Methodik zum Entwick eln und Konstruieren technischer Systeme und Produkte. [Metodología para el desarrollo y construcción de sistemas técnicos y productos], Berlín, Alemania: Beuth Verlag.

Villa, L.F., Jaramillo, M.A., (2005); Desarrollo de software CAD para la industria de la confección, Proyecto de grado ingeniería mecánica, Laboratorio Mecatrónica, Universidad Eafit, Medellín, Colombia. 